\title{
Venous thromboembolism and palliative care
}

\author{
Author: Simon Noble ${ }^{\mathrm{A}}$
}

The management of cancer-associated thrombosis (CAT) is largely informed by data from adequately powered randomised control trials. However, their exclusion criteria have invariably rendered the study populations unrepresentative of those seen day-to-day by palliative care practitioners.

Recent observational data has given insights into the unique challenges of CAT management within the palliative care setting including the natural history of thrombosis in advanced cancer and end-of-life decision making around anticoagulation

Despite developments and some on-going uncertainties, one constant remains; the patient and their experiences. We should, wherever possible, involve them in the decision-making process particularly where the evidence is lacking. By appreciating the values and concerns of our patients, we shall be able to plan care that most meets their needs.

\section{Introduction}

Venous thromboembolism (VTE) comprising of deep vein thrombosis (DVT) and pulmonary embolus (PE) occurs in one in 1,000 adults, but will increase with age, reduced mobility and concurrent chronic illness including cancer. The management of VTE in patients with advanced metastatic cancer and life limiting non-malignant illness has changed considerably, over the past 15 years, from a nihilistic philosophy that a sudden fatal PE should be welcomed, to a more individualised and tailored approach to thrombus prevention and treatment. Challenges of managing VTE in palliative care include; defining the palliative population, recognition of VTE, diagnosis of VTE, treatment of VTE and length of treatment.' This paper shall primarily focus on the current evidence for managing cancer-associated thrombosis (CAT) with particular emphasis on patients with advanced or metastatic disease. It will consider challenges facing clinicians working in palliative care and hospice teams but will also offer guidance to teams involved in supportive care of patients with metastatic disease.

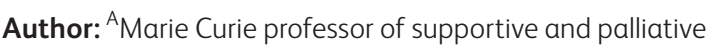
medicine, Cardiff University, Cardiff, UK

\section{Presentation and diagnosis}

Despite a high prevalence of VTE in advanced cancer, CAT is notoriously under reported in hospices and specialist palliative care units (SPCU). In part, this reflects the myriad of pathologies seen in advanced disease, which have presenting symptoms similar to those of VTE. ${ }^{2}$ Dyspnoea is a common symptom and conditions such as anaemia, pulmonary oedema, infection and pleural effusion may be considered before pursuing a diagnosis of PE. Similarly, swollen legs may be attributed to hypoalbuminaemia, left ventricular failure or pelvic lymphadenopathy before considering DVT. Within stand-alone SPCUs/hospices, where access to definitive radiology support requires patient transfer, clinicians may be less likely to pursue and diagnosis of VTE if other pathologies are possible.

While D-dimers are routinely used in the diagnostic work up for VTE, they have little utility in the cancer setting. D-dimers are specific cross-linked fibrin derivatives produced when fibrin is degraded by plasmin. ${ }^{3}$ Concentrations are therefore raised by thrombolysis, making them a highly sensitive indicator for VTE. They

\section{Key points}

The current data supports low molecular weight heparin as the first line management of cancer associated venous thromboembolism.

New data shows that direct-acting oral anticoagulants (DOACs) have a lower rate of venous thromboembolism recurrence but this comes at an expense of major bleeding.

Major bleeding on DOACs is most marked in gastrointestinal and urothelial tumours.

Patients with poor performance status and short prognosis are unlikely to benefit from thromboprophylaxis.

Consider stopping anticoagulation in patients with advanced cancer as death approaches.

KEYWORDS: Cancer associated thrombosis, venous thromboembolism, palliative, hospice, low molecular weight heparin, DOACs 
have a high sensitivity for VTE but low specificity since levels may be raised in the presence of recent surgery, liver disease, cancer, pregnancy and infection. Although D-dimer values are an important exclusory test for the diagnosis of VTE, with a negative predictive value of close to $100 \%$, they have no role in the palliative care setting.

The definitive confirmatory investigation for DVT and PE is compression ultrasonography and computed tomography pulmonary angiography, respectively. ${ }^{4,5}$ Both investigations are relatively easy to access and well tolerated by patients. However, for stand alone hospice and SPCU inpatients, the logistics of organising the tests, which may be undertaken at another institution, may be too difficult for some patients. This has been known to impact on clinicians' threshold for investigating for VTE especially if other causes can be attributed. ${ }^{6}$

\section{Treatment}

While palliative care does not aim to prolong life, it does not aim to shorten it either. There are those of the opinion that 'A large PE may be a nice way to go' implying that a sudden asymptomatic death due to $P E$ is arguably less distressing or burdensome than a prolonged decline due to progressive cancer. However, the concept of fatal PEs being asymptomatic is an erroneous one. A post-mortem study of patients in whom pulmonary embolus was confirmed as the cause of death suggested that a sudden asymptomatic death occurred in only $10 \%$ of patients. The majority have prolonged symptomatic deaths lasting an average of 2 hours, dominated by dyspnoea, tachycardia and distress. ${ }^{7}$

Current clinical guidelines recommend weight adjusted low molecular weight heparin ( $\mathrm{LMWH}$ ) as the first line treatment of CAT, since it has demonstrated superiority over warfarin with respect to preventing recurrent VTE, without an increase in bleeding complications. ${ }^{8}$ In addition, the use of warfarin is particularly challenging in many patients receiving chemotherapy due to drug-drug interactions rendering the international normalised ratio unstable. Conversely, LMWH has fewer drug-drug interactions and rarely requires monitoring.

It is important to note that the trials evaluating warfarin versus LMWH in CAT excluded patients with a life expectancy of less than 3 months, poor performance status, increased bleeding risk, renal impairment, weight $<40 \mathrm{~kg}$, thrombocytopenia and other comorbidities common in palliative patients. Within these randomised trials, the prevalence of metastatic disease, a possible palliative indicator, ranged from approximately 40 to $70 \% .{ }^{9}$ However, several case series of patients under palliative care teams have supported its use in patients with poorer performance status or prognosis.

Since LMWH is administered as a daily subcutaneous injection, there are concerns that this form of administration may have a negative impact on patients' quality of life and be a less acceptable intervention than oral alternatives. Qualitative studies suggest that patients find LMWH acceptable within the context of their cancer journey and quickly adapt to the daily routine of self-injection. ${ }^{10,11}$

\section{Direct-acting oral anticoagulants and cancer}

Two randomised controlled trials have compared a direct-acting oral anticoagulant (DOAC) with LMWH in the treatment of CAT. The Hokusai VTE-cancer study compared 5 days of LMWH followed by edoxaban $60 \mathrm{mg}$ once daily with dalteparin at a dose of $200 \mathrm{IU} / \mathrm{kg}$ for 1 month followed by dalteparin $150 \mathrm{IU} / \mathrm{kg}$ in cancer patients with VTE. ${ }^{12}$ The primary outcome was a composite of recurrent VTE and major bleeding. One-thousand and forty-six patients were recruited. Edoxaban demonstrated non-inferiority with dalteparin with a primary-outcome event in 67 of the 522 patients $(12.8 \%$ ) in the edoxaban group with 71 of the 524 patients $(13.5 \%$ ) in the dalteparin group (hazard ratio $(H R)=0.97 ; 95 \%$ confidence interval $(C I)=0.70-1.36 ; p=0.006$ for non-inferiority; $p=0.87$ for superiority). It appears that edoxaban results in fewer recurrent VTE events, at the expense of more major bleeding episodes. Recurrent VTE occurred in 41 patients (7.9\%) in the edoxaban group and in 59 patients (11.3\%). Major bleeding occurred in 36 patients $(6.9 \%)$ in the edoxaban group and in 21 patients $(4.0 \%)$. Major bleeding was higher in gastrointestinal $(13.1 \%)$ and urothelial $(7.9 \%)$ cancers and it would seem reasonable to avoid DOACs in such cancers.

The SELECT-D study, compared rivaroxaban with dalteparin for the treatment of CAT. ${ }^{13}$ While underpowered, its results were similar to edoxaban. The VTE recurrence rate at 6 months was $11 \%(95 \% \mathrm{CI}=7-17 \%)$ for patients on dalteparin and $4 \%(95 \%$ $\mathrm{CI}=2-9 \%)$ for patients on rivaroxaban. Major bleeds were similar across trial arms but there were more clinically relevant non-major bleeds (CRNMBs) on the rivaroxaban arm; five bleeds (2\%; $95 \%$ $\mathrm{CI}=1-6 \%$ ) on dalteparin compared with 28 bleeds (13\%; $95 \%$ $\mathrm{CI}=9-19 \%)$ on rivaroxaban .

When considering DOACs in the palliative care setting, caution has been advised in the frail and elderly, with underlying hepatic dysfunction, and with impaired renal function (with avoidance of DOACs altogether in those with a creatinine clearance less than $30 \mathrm{~mL} / \mathrm{min}$ ). Another important consideration is the multiple drugdrug interactions that exist with these agents, leading to increased or reduced DOAC plasma levels. While subject to fewer interactions than warfarin, DOACs are particularly sensitive to medicines, which inhibit P-glycoprotein or cytochrome P450 3A4 (CYP3A4). ${ }^{14}$ These include chemotherapies and supportive care medicines commonly used in oncology, which include chemotherapeutic and immunosuppressive agents, hormonal therapies, tyrosine kinase inhibitors and dexamethasone (Table 1).

\section{Challenging scenarios}

Up to $21 \%$ of CAT patients are managed outside of the standard treatment of weight adjusted $\mathrm{LMWH} .{ }^{15}$ These include patients with recurrent VTE despite anticoagulation, patient with thrombocytopenia, and those with bleeding complications. The management of such cases is covered in a guidance document recently published by the International Society for Thrombosis and Haemostasis Scientific Sub-Committee for malignancy and haemostasis, which is summarised in Table $2 .^{16}$

\section{Anticoagulation in the last days of life}

CAT guidelines recommend indefinite anticoagulation for patients with ongoing active cancer but none of them address management of anticoagulation as death approaches. Cancer patients are highly thrombotic at the end of life, most probably due to a combination of disease progression, immobility, dehydration and infection. In a recent case series of 214 patients with CAT who died over a 2-year period, $50 \%$ continued LMWH until death and $11 \%$ up to 7 days prior to 
Table 1. Common drug-drug interactions with direct-acting oral anticoagulants. ${ }^{14}$

\begin{tabular}{|c|c|c|c|c|}
\hline & Dabigatran & Rivaroxaban & Apixaban & Edoxaban \\
\hline \multirow[t]{2}{*}{ Interaction effect } & P-glycoprotein & P-glycoprotein & P-glycoprotein & P-glycoprotein \\
\hline & & CYP3A4 & CYP3A4 & \\
\hline \multirow[t]{6}{*}{ Increases DOAC plasma levels ${ }^{a}$} & & Dasatinib & Dasatinib & \\
\hline & & Imatinib & Imatinib & \\
\hline & Lapatinib & Lapatinib & Lapatinib & Lapatinib \\
\hline & Nilotinib & Nilotinib & Nilotinib & Nilotinib \\
\hline & Sunitinib & Sunitinib & Sunitinib & Sunitinib \\
\hline & Tamoxifen & Tamoxifen & Tamoxifen & Tamoxifen \\
\hline \multirow[t]{4}{*}{ Reduces DOAC plasma levels ${ }^{b}$} & Dexamethasone & Dexamethasone & Dexamethasone & Dexamethasone \\
\hline & Doxorubicin & Doxorubicin & Doxorubicin & Doxorubicin \\
\hline & Enzalutamide & Enzalutamide & Enzalutamide & Enzalutamide \\
\hline & Vinblastine & Vinblastine & Vinblastine & Vinblastine \\
\hline
\end{tabular}

death. ${ }^{17}$ In those who discontinued anticoagulation earlier, there were no reported symptoms attributable to recurrent VTE. Major and CRNMB were experienced by $7 \%$ of those who continued LMWH, to or within 7 days of death. Typically, symptomatic DVT and PE manifest themselves approximately 7 and 21 days, respectively, after the initial pro-thrombotic insult. ${ }^{18}$ If this holds true for patients with advanced cancer, one could stop anticoagulation for patients in whom prognosis is limited to days, thereby minimising bleeding without a recurrence of thrombotic symptoms. If patients develop VTE in the agonal phase, end of life medicines could be used to manage symptoms without risking haemorrhage.

Table 2. Management of challenging cases of cancer-associated thrombosis ${ }^{16}$

Recurrent VTE despite anticoagulation

$>$ If on warfarin, switch to therapeutic LMWH.

$>$ If already on $\mathrm{LMWH}$, increase dose by $25 \%$ or increase back up to therapeutic weight adjusted dose if they are receiving nontherapeutic dosing.

$>$ If no symptomatic improvement use peak anti-Xa level to estimate next dose escalation.

\section{Management of CAT in thrombocytopenia}

$>$ For platelet count $>50 \times 10^{9} \mathrm{~L}^{-1}$ give full therapeutic dose $\mathrm{LMWH}$.

$>$ For acute CAT and platelet count $<50 \times 10^{9} \mathrm{~L}^{-1}$ :

1. full anticoagulation with platelet transfusion to maintain platelet count $>50 \times 10^{9} \mathrm{~L}^{-1}$

2. if platelet transfusion is not possible consider retrievable IVC filter.

$>$ For subacute or chronic CAT and thrombocytopaenia (platelet count $<50 \times 10^{9} \mathrm{~L}^{-1}$ ):

1. reduce therapeutic dose by $50 \%$ or use prophylacitic dose for platelet count $25-50 \times 10^{9} \mathrm{~L}^{-1}$

2. omit LMWH if platelet count $<25 \times 10^{9} \mathrm{~L}^{-1}$.

\section{Bleeding while anticoagulated}

$>$ Assess each bleeding episode to identify bleeding source, severity, impact and reversibility.

$>$ Provide supportive measures to stop bleeding, including transfusion where indicated.

$>$ For a major or life-threatening bleeding episode, withhold anticoagulation:

1. consider IVC filter insertion in patients with acute or subacute CAT with a major or life-threatening bleeding episode

2. do not consider IVC filter insertion in patients with chronic CAT

3. once bleeding resolves, remove retrievable filter (if inserted) and resume/initiate anticoagulation.

CAT = cancer-associated thrombosis; IVC = inferior vena cava; $\mathrm{LMWH}=$ low molecular weight heparin; $\mathrm{VTE}=$ venous thromboembolism. 


\section{Primary thromboprophylaxis}

The prevention of 'hospital acquired thrombosis' is a healthcare priority in many countries, yet remains unclear in SPCUs and hospices. Clinical guidelines suggest that in the absence of contraindications, primary thromboprophylaxis is recommended for acutely ill hospitalised medical patients, including those with underlying cancer. Few SPCUs follow thromboprophylaxis policies and prophylactic LMWH is rarely initiated or continued on admission. Reasons for not following guidelines, include a view that VTE is not a particular problem and that the studies which inform prophylaxis were conducted in an unrepresentative population using outcome measures of limited utility in the hospice or SPCU. ${ }^{19}$

A recent multicentre observational study of 1,199 patients (90\% of whom had a cancer diagnosis) admitted to 22 hospices/SPCUs demonstrated a low incidence of VTE but a high incidence of clinically relevant bleeding $(9.8 \%)$. Multivariate analysis identified that bleeding was associated with thromboprophylaxis ( $p=0.04$, $H R=1.48(1.02-2.15)$ ), suggesting that the bleeding risks of VTE prophylaxis might outweigh the benefits in this population. ${ }^{20}$

The Hospice Inpatient Deep Vein Thrombosis Detection study has recently been published and further challenges the perceived benefit of pharmacological thromboprophylaxis. ${ }^{21}$ In this prospective longitudinal observational study, 343 cancer patients underwent bilateral femoral vein ultrasonography on admission and weekly until death or discharge. Patients were excluded if admitted for terminal care (estimated prognosis $<5$ days). Patients had a mean Australian-modified Karnofsky Performance Score of 49 and survival of 44 days, indicating a highly dependent and poor prognosis cohort. Femoral DVT was observed in $28 \%$ of participants with minimal symptoms and there was no difference in survival in those with or without DVT. These data challenge the utility of thromboprophylaxis in patients of poor performance status and prognosis although its role in selected patients of good performance status, with temporary elevations of thrombotic risk factors remains to be clarified.

\section{Conclusion}

As people live longer with metastatic disease and receive palliative chemotherapies into older age, there has been a blurring around the margins of what constitutes palliative, supportive and end of life care when managing venous thromboembolism. Cancer associated thrombosis will continue to be challenge for any team looking after patients with advanced metastatic cancer and life limiting non-malignant illness. With the increasing heterogeneity of malignant disease, breadth of anticancer treatments and available anticoagulants it is highly unlikely that a one size fits all approach to CAT management will exist in the future. Just as anticancer treatments have become so individualised that their indications may be based upon molecular biomarkers, it is reasonable to expect other aspects of cancer management to be individualised, particularly when considering advanced disease where quality of life takes primacy over longevity.

\section{References}

1 Noble S. The challenges of managing cancer related venous thromboembolism in the palliative care setting. Postgraduate medical journal 2007;83:671-4.
2 Noble S, Pasi J. Epidemiology and pathophysiology of cancerassociated thrombosis. Br J Cancer 2010;102(Suppl 1):S2-9.

3 Kelly J, Hunt B]. Role of D-dimers in diagnosis of venous thromboembolism. Lancet 2002;359:456-8.

4 Fraser JD, Anderson DR. Deep venous thrombosis: recent advances and optimal investigation with US. Radiology 1999;211:9-24.

5 Remy-Jardin M, Remy J, Deschildre F et al. Diagnosis of pulmonary embolism with spiral CT: comparison with pulmonary angiography and scintigraphy. Radiology 1996;200:699-706.

6 Sheard L, Prout H, Dowding D et al. Barriers to the diagnosis and treatment of venous thromboembolism in advanced cancer patients: a qualitative study. Palliative medicine 2013;27:339-48.

7 Havig O. Deep vein thrombosis and pulmonary embolism. An autopsy study with multiple regression analysis of possible risk factors. Acta Chir Scand Supp/ 1977;478:1-120.

8 Farge D, Debourdeau P, Beckers $\mathrm{M}$ et al. International clinical practice guidelines for the treatment and prophylaxis of venous thromboembolism in patients with cancer. J Thromb Haemost 2013;11:56-70.

9 Noble S, Sui J. The treatment of cancer associated thrombosis: does one size fit all? Who should get LMWH/warfarin/DOACs? Thromb Res 2016;140(Suppl 1):S154-9.

10 Seaman S, Nelson A, Noble S. Cancer-associated thrombosis, lowmolecular-weight heparin, and the patient experience: a qualitative study. Patient Prefer Adherence 2014;8:453-61.

11 Noble S, Prout H, Nelson A. Patients' Experiences of LIving with CANcer-associated thrombosis: the PELICAN study. Patient Prefer Adherence 2015;9:337-45.

12 Raskob GE, van Es N, Verhamme P et al. Edoxaban for the treatment of cancer-associated venous thromboembolism. N Engl J Med 2018;378:615-24.

13 Young AM, Marshall A, Thirlwall ] et al. Comparison of an oral factor Xa inhibitor with low molecular weight heparin in patients with cancer with venous thromboembolism: Results of a Randomized Trial (SELECT-D). J Clin Oncol 2018;36:2017-23.

14 Lee AY, Peterson EA. Treatment of cancer-associated thrombosis. Blood 2013;122:2310-7.

15 Noble S, Pease N, Sui ] et al. Impact of a dedicated cancer-associated thrombosis service on clinical outcomes: a mixed-methods evaluation of a clinical improvement exercise. BMJ open 2016;6:e013321.

16 Carrier M, Khorana AA, Zwicker ] et al. Management of challenging cases of patients with cancer-associated thrombosis including recurrent thrombosis and bleeding: guidance from the SSC of the ISTH. J Thromb Haemost 2013;11:1760-5.

17 Noble S, Banerjee S, Pease N. Anticoagulation for cancer associated thrombosis at the end of life: review of a case series of 214 patients. Palliat Med 2018;32(1S):47-8.

18 Hunt B]. The prevention of hospital-acquired venous thromboembolism in the United Kingdom. Br J Haematol 2009;144:642-52.

19 Noble S, Nelson A, Finlay IG. Factors influencing hospice thromboprophylaxis policy: a qualitative study. Palliat Med 2008;22:808-13.

20 Tardy B, Picard S, Guirimand F et al. Bleeding risk of terminally ill patients hospitalized in palliative care units: the RHESO study. J Thromb Haemost 2017:15:420-8.

21 White C, Noble S, Watson M et al. Prevalence, symptom burden, and natural history of deep vein thrombosis in people with advanced cancer in specialist palliative care units (HIDDen): a prospective longitudinal observational study. Lancet Haematol 2019;6:e79-88.

Address for correspondence: Prof Simon Noble, Marie Curie Palliative Care Research Centre, 8th Floor, Neuadd Meirionnydd, Cardiff University, Heath Park Campus, Cardiff CF14 4YS, UK.

Email: noblesi1@cardiff.ac.uk 